Classification

Physics Abstracts

$86.10 \mathrm{~K}$

\title{
Etude théorique et expérimentale d'un capteur solaire plan à air en régime dynamique
}

\author{
T. Letz and M. Lallemand
}

I.N.S.A., Laboratoire d'Energétique et d'Automatique, 69621 Villeurbanne, France

\begin{abstract}
Résumé. - Le travail présenté concerne l'analyse du comportement d'un capteur solaire plan à air fonctionnant en régime dynamique. Un modèle basé sur une résolution des équations de bilans thermiques par une méthode nodale a été établi. Sa validation est réalisée à partir d'essais effectués en ensoleillement naturel et en ensoleillement artificiel. L'écart absolu entre les rendements théoriques et expérimentaux est inférieur à $3 \%$. La sensibilité du rendement à différents paramètres, accessibles ou non par l'expérience, a été étudiée ; en particulier il s'agit de la sélectivité de l'absorbeur, de l'importance des fuites d'air, et des conditions de fonctionnement du capteur.
\end{abstract}

\begin{abstract}
This paper reports a transient study of a solar flat-plate air collector. A time-dependent heat transfer model has been developed. The heat balance equations are solved by a multi-node method. Results are compared with indoor and outdoor tests. They are in good agreement with experimental data. The effects of various parameters, measured or not, on the collector efficiency have been studied. In particular, the use of a selective coating, the effects of air leaks or the influence of operating conditions have been investigated.
\end{abstract}

\section{Introduction.}

Très répandu aux Etats-Unis, le chauffage aéraulique est moins fréquent en France. Pourtant, il semble que cette technique puisse être utilisée avec profit dans des installations solaires à capteur à air, puisque l'air chauffé peut être envoyé directement dans les locaux. Il importe alors de bien connaître les caractéristiques de fonctionnement des capteurs dans des conditions variables. De nombreuses études expérimentales ont été réalisées avec des capteurs solaires à air de géométries diverses $[1,2]$.

Le capteur modulaire étudié, produit industriellement, permet de réaliser des toitures captatrices de dimensions très variées en vue du chauffage des habitations [3]. Pour tester ce capteur solaire, des essais ont été effectués en ensoleillement naturel et sous simulateur solaire. La deuxième méthode permet de travailler dans des conditions plus stables et reproductibles. En effet, en site extérieur, les conditions météorologiques ne sont pas fixes et il est difficile d'obtenir un régime permanent du fonctionnement du capteur. Le comportement du capteur testé expérimentalement a été modélisé en régime dynamique en utilisant une discrétisation nodale. L'air circule d'abord dans le canal 2 avant de s'écouler dans le canal 1.

\section{Expérimentation.}

2.1 INSTALLATIONS EXPÉRIMENTALES ET ESSAIS. Le capteur solaire est du type plan, composé de modules de surface unitaire $0,405 \mathrm{~m}^{2}(0,45 \times 0 ; 9)$ dont une coupe est représentée sur la figure 1 .
Chaque module est monté sur un panneau de particules constituant la cloison inférieure de la lame d'air dynamique. Celle-ci, limitée par l'absorbeur et cette cloison, est séparée en deux lames parallèles pour permettre une double circulation de l'air. L'absorbeur, en alliage d'aluminium, est recouvert d'un revêtement sélectif $(\varepsilon=0,15, \alpha=0,91)$. Le

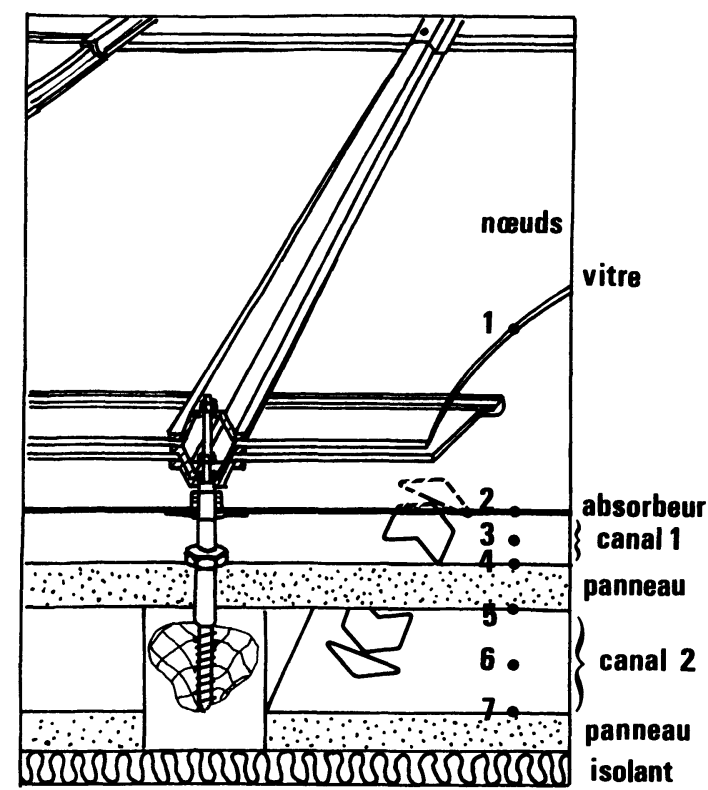

Fig. 1. - Schéma du capteur étudié.

[Schematic view of experimental collector.] 
capteur comporte un seul vitrage d'épaisseur $3 \mathrm{~mm}$. Un circuit aéraulique permet d'alimenter le capteur avec des débits d'air variables et de fixer la température d'entrée de l'air dans le capteur. La circulation de l'air est assurée par un ventilateur placé en aval du capteur afin que celui-ci soit légèrement en dépression et que les fuites d'air se produisent de l'extérieur vers l'intérieur.

Les essais en ensoleillement naturel ont été réalisés à Lyon, au mois de juillet, avec un capteur orienté face au Sud et incliné à $45^{\circ}$. Pour chaque essai, la température de l'air à l'entrée du capteur ainsi que le débit d'air sont fixés. Le rendement du système évolue alors sur une journée en fonction des conditions extérieures (ensoleillement, température, vent, ...).

Des essais sont également effectués avec un simulateur solaire permettant de simuler des niveaux d'éclairement dans le plan du capteur compris entre 200 et $1000 \mathrm{~W} / \mathrm{m}^{2}$ et orientable pour permettre d'éclairer le capteur d'inclinaison variable en gardant le plan des projecteurs parallèle au plan du capteur. Il est constitué de 23 lampes à incandescence dont la répartition est déterminée de façon à obtenir un éclairement homogène dans le plan du capteur (écart inférieur à $10 \%$ ) [3]. Par rapport au spectre solaire, le spectre des lampes est décalé vers l'infrarouge. L'éclairement infrarouge reçu par le capteur est obtenu par différence entre le rayonnement total mesuré par un pyradiomètre et le rayonnement global mesuré par un pyranomètre. Ce rayonnement infrarouge de grande longueur d'onde est dû à l'échauffement des lampes et au rayonnement propre du local. L'éclairement infrarouge en provenance de la pièce, calculé en assimilant celle-ci à un corps noir, est retranché à la valeur mesurée pour obtenir l'éclairement dû au simulateur solaire. Ce dernier est donné dans le tableau I pour différentes valeurs de l'éclairement global. Dans la modélisation, ce rayonnement infrarouge est pris en compte pour avoir des résultats théoriques en bon accord avec l'expérience.

Tableau I. - Eclairement infrarouge dî au simulateur solaire.

[Infrared radiation due to the solar simulator].

\begin{tabular}{l|c|c|c}
$\begin{array}{c}\text { Eclairement global mesuré } \\
\left(\mathrm{W} / \mathrm{m}^{2}\right)\end{array}$ & 818 & 650 & 462 \\
\hline $\begin{array}{c}\text { Eclairement infrarouge des } \\
\text { lampes }\left(\mathrm{W} / \mathrm{m}^{2}\right)\end{array}$ & 190 & 178 & 144
\end{tabular}

2.2 RÉsultats. - Le rendement du capteur est défini comme le rapport de la puissance utile $\dot{Q}_{u}$ extraite du capteur au flux solaire incident $G$. La puissance utile est évaluée à l'aide d'un bilan enthalpique effectué en tenant compte des fuites d'air, mesurées expérimentalement :

$$
\dot{Q}_{\mathrm{u}}=\dot{M}_{\mathrm{s}} h_{\mathrm{s}}-\dot{M}_{\mathrm{f}} h_{\mathrm{a}}-\dot{M}_{\mathrm{e}} h_{\mathrm{e}}
$$

où $\dot{M}$ est le débit massique en entrée (e) ou sortie (s) et pour les fuites (f) et $h$ l'enthalpie massique de l'air (indice $\mathrm{a}=$ ambiance). Si l'on exprime l'enthalpie de l'air considéré comme humide en fonction de sa température et de son humidité absolue $x$, le rendement est donné par la relation:

$$
\eta=\frac{c_{\mathrm{p}} \dot{V}_{\mathrm{s}}(1+1,88 x)\left[\left(T_{\mathrm{s}}-T_{\mathrm{e}}-\tau_{\mathrm{f}}\left(T_{\mathrm{a}}-T_{\mathrm{e}}\right) T_{\mathrm{s}} / T_{\mathrm{a}}\right)\right]}{v_{\mathrm{s}} \cdot G \cdot S}
$$

$S$ est la surface du capteur, $c_{\mathrm{p}}$ la chaleur massique de l'air et $v$ son volume massique. Le taux de fuite $\tau_{\mathrm{f}}$ est le rapport du débit volumique des fuites sur le débit volumique $\dot{V}_{\mathrm{s}}$ de l'air à la sortie.

La figure 2 montre l'évolution du rendement instantané du capteur et de l'éclairement global dans le plan du capteur en ensoleillement naturel. Pour cet essai, la température de l'air à l'entrée du capteur vaut environ $40^{\circ} \mathrm{C}$ et le débit est de 75 $\mathrm{m}^{3} / \mathrm{h} . \mathrm{m}^{2}$. Le rendement maximum est atteint entre 14 et $15 \mathrm{~h}$ alors que le flux solaire est maximum à midi. Ce décalage est dû à la superposition de deux effets. L'augmentation de la température ambiante au cours de la journée entraîne une diminution des pertes thermiques du capteur et contribue à augmenter le rendement. Mais la raison principale est l'influence de l'inertie élevée du capteur, due à la masse importante de bois située à l'intérieur de l'isolant. Le capteur accumule de l'énergie le matin lorsque le flux augmente et la restitue l'après-midi lorsque le flux décroît.

Il n'est pas possible en ensoleillement naturel de



Fig. 2. - Evolution du rendement au cours d'une journée. [Hourly variations of the collector efficiency.]

tracer des courbes donnant l'évolution du rendement en fonction d'un paramètre en fixant les autres, car plusieurs varient simultanément (flux solaire, tempé- 
Tableau II. - Comparaison théorie-expérience en ensoleillement naturel.

[Comparison of theoretical and experimental collector efficiencies for outdoor conditions.]

\begin{tabular}{l|c|c|c|c|c|c}
\multicolumn{1}{c|}{ Numéro de l'essai } & 1 & 2 & 3 & 4 & 5 & 6 \\
\hline Température ambiante $\left({ }^{\circ} \mathrm{C}\right)$ & 23,0 & 26,0 & 33,4 & 29,9 & 23,8 & 25,5 \\
\hline Vitesse du vent $(\mathrm{m} / \mathrm{s})$ & 2,0 & 0,8 & 0,2 & 2,0 & 1,5 & 1,0 \\
\hline Humidité relative $(\%)$ & 33 & 36 & 36 & 35 & 32 & 36 \\
\hline Eclairement global $\left(\mathrm{W} / \mathrm{m}^{2}\right)$ & 785 & 862 & 804 & 853 & 902 & 884 \\
\hline Débit à la sortie $\left(\mathrm{m}^{3} / \mathrm{h} \cdot \mathrm{m}^{2}\right)$ & 74 & 74 & 74 & 74 & 110 & 31 \\
\hline Taux de fuite $(\%)$ & 5,7 & 5,7 & 5,7 & 5,7 & 5,9 & 3,9 \\
\hline Température d'entrée $\left({ }^{\circ} \mathrm{C}\right)$ & 24,2 & 40,2 & 50,0 & 60,8 & 38,0 & 40,5 \\
\hline Température de ciel $\left({ }^{\circ} \mathrm{C}\right)$ & 3,1 & 7,5 & 16,3 & 11,8 & 3,8 & 6,9 \\
\hline Température de sortie mesurée $\left({ }^{\circ} \mathrm{C}\right)$ & 39,0 & 54,8 & 62,8 & 70,2 & 49,0 & 65,4 \\
\hline Température de sortie calculée $\left({ }^{\circ} \mathrm{C}\right)$ & 39,3 & 53,9 & 62,2 & 69,4 & 48,9 & 62,5 \\
\hline Rendement mesuré $(\%)$ & 43,8 & 39,5 & 36,9 & 28,0 & 44,0 & 25,6 \\
\hline Rendement calculé $(\%)$ & 44,8 & 37,6 & 35,9 & 26,4 & 43,7 & 23,1
\end{tabular}

rature...). Cependant des essais ont été effectués à débit et température d'entrée d'air variables. Les rendements donnés dans le tableau II sont mesurés au voisinage du midi solaire lorsque le flux varie peu. On constate une diminution du rendement lorsque la température de l'air à l'entrée du capteur augmente (essais $1,2,3,4$ ) ou lorsque le débit diminue (essais $5,2,6$ ), les autres paramètres restant à peu près constants.

En ensoleillement artificiel, chaque courbe est obtenue en faisant varier un paramètre tout en donnant aux autres une valeur constante (débit d'air : $70 \mathrm{~m}^{3} / \mathrm{h} . \mathrm{m}^{2}$; température d'entrée : $25^{\circ} \mathrm{C}$; éclairement : $800 \mathrm{~W} / \mathrm{m}^{2}$; inclinaison de capteur : $60^{\circ}$ ). Pour tous ces essais, le seul paramètre non parfaitement maîtrisé est la température ambiante ; sa plage de variation est comprise entre 20 et $23^{\circ} \mathrm{C}$. La figure 3 montre l'influence du débit volumique et de l'inclinaison du capteur. Le rendement augmente en fonction du débit volumique car la vitesse de l'air léchant l'absorbeur est plus grande et donc l'échange thermique est amélioré. Cependant à partir d'un débit de $100 \mathrm{~m}^{3} / \mathrm{h} . \mathrm{m}^{2}$ correspondant à des vitesses dans la lame dynamique d'environ $2 \mathrm{~m} / \mathrm{s}$, la courbe tend vers une limite. Donc, il ne sert à rien d'utiliser un débit trop important, et cela d'autant plus qu'un débit trop fort conduit à une température de sortie de l'air basse, qui rend difficile l'utilisation de l'énergie produite. L'influence de l'inclinaison du capteur sur le rendement est faible. Toutefois, lorsque le capteur est redressé, il augmente légère-

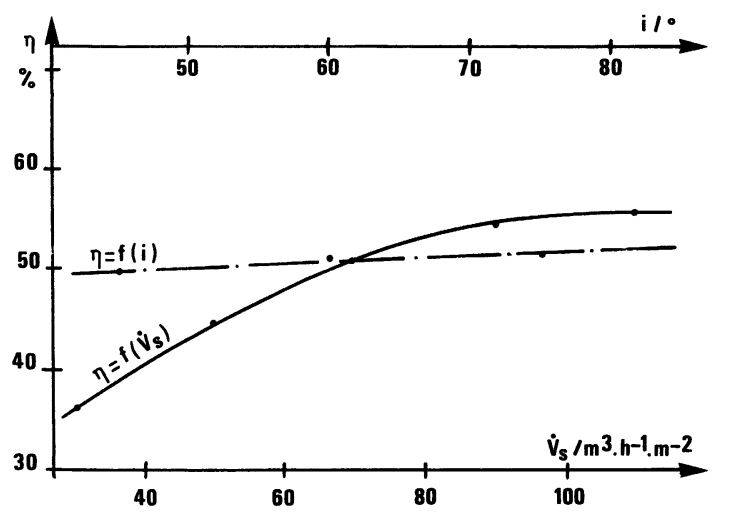

Fig. 3. - Influence de l'inclinaison du capteur et du débit volumique de l'air sur le rendement.

[Effects of collector tilt angle and outlet flow rate.]

ment en raison d'un échauffement moindre de la vitre qui limite les déperditions de chaleur. En effet, Hollands [4] a constaté une diminution des échanges par convection naturelle dans la lame d'air statique entre la vitre et l'absorbeur lorsque l'inclinaison du capteur croît.

La figure 4 met en évidence l'influence de la température d'entrée d'air dans le capteur et de l'éclairement. Le rendement diminue lorsque la température d'entrée d'air croît en raison de l'augmentation des pertes thermiques du capteur. On voit donc l'intérêt pour un capteur à air, utilisé 


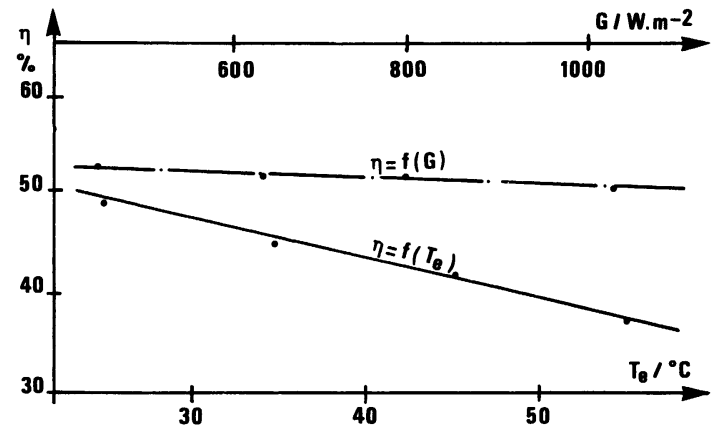

Fig. 4. - Influence de la température d'entrée d'air et de l'éclairement sur le rendement.

[Effects of inlet air temperature and intensity of solar radiation.]

pour le chauffage des locaux, de travailler en chauffage direct, c'est-à-dire d'avoir une température d'entrée d'air égale ou inférieure à la température des locaux. Une autre utilisation intéressante est le préchauffage de l'air de renouvellement des locaux lorsque ce type de déperditions est important. En ensoleillement artificiel, le rendement semble n'être que très peu sensible à la variation de l'éclairement.

\section{Méthode de calcul du rendement instantané en régime dynamique.}

Pour modéliser le comportement du capteur solaire en régime dynamique, on le discrétise fictivement par un certain nombre de nœuds. Les équations du régime dynamique sont obtenues en écrivant les bilans énergétiques pour chaque nœud. $\mathrm{Ce}$ système d'équations est résolu par la méthode des variables d'état.

3.1 HYPOTHÈSES DE CALCUL. - Les paramètres influençant le fonctionnement du capteur sont de deux natures différentes :

- paramètres intrinsèques : ce sont soit des caractéristiques propres du capteur, soit des caractéristiques du fluide caloporteur qui le traverse,

- paramètres extrinsèques : ils regroupent l'ensemble des conditions climatiques dont dépend le fonctionnement du capteur. L'éclairement incident est séparé en un éclairement de longueur d'onde inférieure à $2,5 \mu \mathrm{m}$ et un éclairement infrarouge de longueur d'onde supérieure à $2,5 \mu \mathrm{m}$. Ceci permet de tenir compte du rayonnement du ciel lorsque le capteur est placé à l'extérieur ou de celui du local d'essais lors des essais avec simulateur solaire.

La modélisation du capteur est basée sur une discrétisation nodale de celui-ci faisant apparaître 7 nœuds. Les nœuds $1,2,4,5$ et 7 correspondent à cinq éléments du capteur supposés homogènes en température (Fig. 1): la vitre (1), l'absorbeur (2), les parties supérieure et inférieure du panneau de particules intermédiaire $(4,5)$ et le panneau arrière (7). Les nœuds 3 et 6 représentent respectivement les lames d'air dynamique supérieure et inférieure. Le gradient thermique dans l'épaisseur des éléments est négligé sauf pour le panneau intermédiaire. Les pertes thermiques latérales sont intégrées aux pertes arrières. Les fuites d'air sont uniformément réparties le long de l'absorbeur.

\subsection{BILANS THERMIQUES EN RÉGIME DYNAMIQUE.}

- Pour chaque nœud i, de capacité calorifique $C_{\mathrm{i}}$, un bilan thermique est effectué tenant compte des échanges radiatifs, conductifs et convectifs avec les autres nœuds et avec le milieu extérieur (indices ci pour le ciel et av ou ar s'il s'agit de l'ambiance à l'avant ou à l'arrière du capteur).

Vitre (nœud 1)

$$
\begin{aligned}
C_{1} \dot{T}_{1}= & S_{2}\left(h_{12}+h_{12}^{\prime}\right)\left(T_{2}-T_{1}\right)+S_{1} \alpha_{1} G+ \\
& +S_{1} h_{1 \mathrm{av}}\left(T_{\mathrm{av}}-T_{1}\right)+S_{1} h_{1 \mathrm{ci}}^{\prime}\left(T_{\mathrm{ci}}-T_{1}\right)
\end{aligned}
$$

$h_{\mathrm{ij}}$ et $h_{\mathrm{ij}}^{\prime}$ sont les coefficients d'échange convectif et radiatif entre les nœuds $\mathrm{i}$ et $\mathrm{j} . \alpha_{1} G$ représente la fraction de l'éclairement solaire global absorbée par la vitre. $\dot{T}_{\mathrm{i}}$ est la dérivée par rapport au temps de la température $T_{\mathrm{i}}$.

Absorbeur (nœud 2)

$$
\begin{aligned}
C_{2} \dot{T}_{2}= & S_{2}\left(h_{12}+h_{12}^{\prime}\right)\left(T_{1}-T_{2}\right)+S_{2} \beta G+ \\
& +S_{2} h_{23}\left(T_{3}-T_{2}\right)+S_{2} h_{24}^{\prime}\left(T_{4}-T_{2}\right)
\end{aligned}
$$

$S_{2} \beta G$ représente la puissance absorbée par la surface éclairée de l'absorbeur.

Air circulant dans le canal 1 (nœud 3)

$$
\begin{aligned}
C_{3} \dot{T}_{3} & =S_{2} h_{23}\left(T_{2}-T_{3}\right)+S_{4} h_{43}\left(T_{4}-T_{3}\right)+ \\
& +\dot{M}_{\mathrm{c}} c_{\mathrm{p} 3}\left(T_{\mathrm{i}}-T_{\mathrm{s}}\right)+\dot{M}_{\mathrm{s}} \tau_{\mathrm{f}} c_{\mathrm{p} 3}\left(T_{\mathrm{av}}-T_{\mathrm{s}}\right)
\end{aligned}
$$

$T_{\mathrm{i}}$ et $T_{\mathrm{s}}$ sont les températures de l'air respectivement à l'entrée et à la sortie du canal 1 , telles que :

$$
T_{\mathrm{i}}=2 T_{6}-T_{\mathrm{e}} \text { et } T_{\mathrm{s}}=2\left(T_{3}-T_{6}\right)+T_{\mathrm{e}} .
$$

Pour le canal 2, représenté par le nœud 6, les termes qui interviennent dans le bilan sont du même type.

Partie supérieure du panneau de particules intermédiaire (nœud 4)

$$
\begin{array}{r}
C_{4} \dot{T}_{4}=S_{4} h_{24}^{\prime}\left(T_{2}-T_{4}\right)+S_{4} h_{43}\left(T_{3}-T_{4}\right)+ \\
+S_{7}\left(\lambda_{\mathrm{p}} / e_{\mathrm{p}}\right)\left(T_{5}-T_{4}\right) .
\end{array}
$$

L'équation du bilan pour le nœud 5 est du même type.

\section{Panneau arrière (nœud 7)}

$$
\begin{aligned}
C_{7} \dot{T}_{7}=S_{7} h_{67}\left(T_{6}-T_{7}\right) & +S_{7} h_{57}^{\prime}\left(T_{5}-T_{7}\right)+ \\
& +U_{\text {ar }} S_{\text {ar }}\left(T_{\text {ar }}-T_{7}\right) .
\end{aligned}
$$


Le dernier terme représente la puissance perdue par la face arrière du capteur. Le coefficient de pertes thermiques à l'arrière du capteur $U_{\mathrm{ar}}$ a été évalué expérimentalement.

Le rendement est alors déterminé à partir de la relation (2).

\subsection{EVALUATION DES COEFFICIENTS D'ÉCHANGE.}

3.3.1 Coefficient d'échange convectif entre la vitre et l'air ambiant. - Pour les essais en l'absence de vent, Kreith et Kreider [4] donnent une expression du coefficient d'échange en convection naturelle $h\left(\mathrm{~W} / \mathrm{m}^{2} \mathrm{~K}\right)$, entre l'air ambiant et un plan de longueur $L$ et d'inclinaison $i$ :

$$
\begin{aligned}
& \text { en régime laminaire }: h=1,42\left[\frac{\left(T_{1}-T_{\mathrm{av}}\right) \sin i}{L}\right]^{0,25} \\
& \text { en régime turbulent }: h=0,95\left[\left(T_{1}-T_{\mathrm{av}}\right) \sin i\right]^{0,25} \text {. }
\end{aligned}
$$

Pour un capteur situé à l'extérieur, en présence d'un vent de vitesse $V(\mathrm{~m} / \mathrm{s}), h\left(\mathrm{~W} / \mathrm{m}^{2} \mathrm{~K}\right)$ est donné par la relation [5] :

$$
h=5,7+3,8 \mathrm{~V} \text {. }
$$

3.3.2 Coefficient d'échange convectif entre la vitre et l'absorbeur. - Parmi les nombreuses corrélations donnant le nombre de Nüsselt $\mathrm{Nu}_{\mathrm{i}}$, calculé pour diverses inclinaisons $i$ et différentes valeurs du nombre de Grashof $G r$, celle retenue pour $0^{\circ}<i<75^{\circ}$ est la corrélation de Hollands [4] :

$$
N u_{i}=1+1,44\left[1-\frac{1708}{R a \cos i}\right]^{*}\left[1-\frac{1708(\sin 1,8 i)^{1,6}}{R a \cos i}\right]+\left[\left(\frac{R a \cos i}{5830}\right)^{1 / 3}-1\right]^{*} \text {. }
$$

Dans cette expression $R a$ est le nombre de Rayleigh et l'astérisque indique que la quantité entre crochets est prise égale à 0 pour toute valeur négative. Pour $i>75^{\circ}$, la corrélation utilisée [6] s'écrit :

$$
N u_{i}=(G r / 4085)^{0,3} .
$$

3.3.3 Coefficient d'échange par convection forcée. Les corrélations utilisées pour calculer le coefficient d'échange dans la lame d'air dynamique sont celles de Hausen [7] pour les régimes laminaire et transitoire et celle de Tan et Charters [8] en régime turbulent.

3.3.4 Coefficient d'échange radiatif. - Le coefficient d'échange radiatif $h_{\mathrm{ij}}^{\prime}$ correspondant aux échanges entre deux plans parallèles calculés en utilisant les températures et les émissivités $\varepsilon_{\mathrm{i}}$ et $\varepsilon_{\mathrm{j}}$ des surfaces en regard, est donné par la relation :

$$
h_{\mathrm{ij}}^{\prime}=\frac{\sigma\left(T_{\mathrm{i}}^{2}+T_{\mathrm{j}}^{2}\right)\left(T_{\mathrm{i}}+T_{\mathrm{j}}\right)}{\frac{1}{\varepsilon_{\mathrm{i}}}+\frac{1}{\varepsilon_{\mathrm{j}}}-1}
$$

avec $\sigma=$ constante de Stefan-Boltzmann. Sur un intervalle de calcul, la linéarisation des pertes radiatives entraîne une erreur relative inférieure à $0,5 \%$ dans le cas où l'écart entre $T_{\mathrm{i}}$ et $T_{\mathrm{j}}$ est maximum.

3.4 MÉTHOde DE RÉSOlution. - Les sept équations de bilans, établies pour chaque nœud, sont écrites sous la forme d'un système matriciel continu :

$$
\dot{\mathbf{T}}=\mathbf{A}(t) \cdot \mathbf{T}+\mathbf{B}(t) \cdot \mathbf{E}
$$

dans lequel T est le vecteur d'état formé par les températures des 7 nœuds, $\mathbf{T}$ est le vecteur formé par les dérivées par rapport au temps de ces températures, $\mathbf{A}(t)$ est une matrice à 7 lignes et 7 colonnes, $\mathbf{B}(t)$ une matrice à 7 lignes et 5 colonnes, et $\mathbf{E}$ est le vecteur des sollicitations du système tel que :

$$
\mathbf{E}=\left[\begin{array}{l}
G \\
T_{\mathrm{ci}} \\
T_{\mathrm{av}} \\
T_{\mathrm{ar}} \\
T_{\mathrm{e}}
\end{array}\right] .
$$

Les coefficients des matrices $\mathbf{A}(t)$ et $\mathbf{B}(t)$ sont des fonctions du temps, car ils dépendent des coefficients d'échange $h$ et $h^{\prime}$, eux-mêmes fonction des températures variables avec le temps. Pour de petites variations de ces températures, les coefficients d'échange sont considérés comme constants, ainsi que les coefficients des matrices $\mathbf{A}$ et $\mathbf{B}$. Nous pouvons donc faire l'approximation que l'équation (8) est celle d'un système linéaire stationnaire pendant des durées telles que les variations des températures ne dépassent pas un seuil fixé à $1^{\circ} \mathrm{C}$.

L'équation continue (8) peut être transformée en une équation d'état discrète de la forme [9]:

$$
\mathbf{T}_{k+1}=\mathbf{M}(P e) \cdot \mathbf{T}_{k}+\mathbf{N}(P e) \cdot \mathbf{E}_{k}
$$

avec

$\mathbf{T}_{k}$ : vecteur des températures au temps $k . P e$

$\mathbf{E}_{k}$ : vecteur des sollicitations au temps $k . P e$

$P e$ : période d'échantillonnage. 
Les matrices $\mathbf{M}(P e)$ et $\mathbf{N}(P e)$ sont définies par:

$$
\begin{aligned}
& \mathbf{M}(P e)=\exp (\mathbf{A} \cdot P e) \\
& \mathbf{N}(P e)=\int_{0}^{P e} \exp (\mathbf{A} \cdot v) \cdot \mathbf{B} \cdot \mathrm{d} v .
\end{aligned}
$$

Pour effectuer les simulations, la période d'échantillonnage $P e$ est fixée à $2 \mathrm{~s}$, ce qui réalise un compromis acceptable entre le temps nécessaire au calcul de $\mathbf{M}$ et $\mathbf{N}$ et le temps nécessaire au calcul du vecteur d'état par la relation (9). Les matrices $\mathbf{A}, \mathbf{B}$, $\mathbf{M}$ et $\mathbf{N}$ sont actualisées périodiquement, à moins qu'une variation importante des températures n'impose plus rapidement un nouveau calcul de celles-ci.

\section{Validation du modèle.}

Les variables d'entrée du modèle sont les températures ambiantes devant et derrière le capteùr, la vitesse du vent, l'humidité absolue, l'éclairement global dans le plan du capteur, la pression atmosphérique, la température de ciel, l'inclinaison du capteur, le débit volumique à la sortie, le taux de fuite et la température d'entrée de l'air. Les résultats obtenus expérimentalement sont comparés avec ceux obtenus par le modèle pour lequel les onze paramètres d'entrée sont ceux mesurés au cours de l'expérience.

4.1 VALIDATION EN ENSOLEILLEMENT NATUREL. - La température de ciel, qui caractérise les échanges radiatifs entre le capteur et son environnement, n'est pas mesurée. Cette température est essentiellement fonction de la température de l'air extérieur et de son humidité. Pour l'évaluer, la corrélation de Berhadl-Fromberg [10] est utilisée :

$$
T_{\mathrm{ci}}=T_{\mathrm{a}}\left(0,741+0,0062 P_{\mathrm{v}}\right)^{0,25} .
$$

Elle donne la température de ciel en fonction de la température ambiante et de la pression de vapeur d'eau $P_{\mathrm{v}}$ (mbar), en l'absence de toute nébulosité.

Dans le tableau II sont comparés les résultats théoriques et expérimentaux pour les six essais décrits dans le paragraphe 2.2. La comparaison est faite en régime statique. Les résultats théoriques et expérimentaux sont très proches, sauf pour les débits faibles, puisque l'écart absolu entre les rendements mesurés et calculés ne dépasse pas $3 \%$ et que les températures de sortie mesurées et calculées ne diffèrent pas de plus de $1 \mathrm{~K}$.

4.2 VALIDATION EN RÉGIME DYNAMIQUE, SOUS SIMULATEUR SOLAIRE. - Le simulateur solaire émettant beaucoup d'infrarouge, à cause de l'échauffement des lampes et des réflecteurs, il est nécessaire de tenir compte de ce rayonnement $\phi_{\text {IR }}$ (Tableau I) qui s'ajoute au rayonnement de grande longueur d'onde émis par la pièce à la température $T_{\mathrm{a}}$. La température de ciel intègre ces deux composantes :

$$
T_{\mathrm{ci}}=\sqrt[4]{\frac{\phi_{\mathrm{IR}}+\sigma T_{\mathrm{a}}^{4}}{\sigma}} .
$$

Pour valider le modèle en régime dynamique, les résultats expérimentaux obtenus avec un échelon d'éclairement de $800 \mathrm{~W} / \mathrm{m}^{2}$ sont comparés avec les valeurs données par une simulation dans les mêmes conditions. La comparaison est faite sur les températures de l'air en sortie du capteur et sur les rendements (Fig. 5).



Fig. 5. - Comparaison théorie-expérience en régime dynamique.

[Comparison of theoretical and experimental collector performance.]

Les points expérimentaux sont très proches de la courbe théorique et les écarts entre les températures mesurées et calculées ne dépassent pas $0,5 \mathrm{~K}$, ce qui conduit à un écart absolu sur le rendement inférieur à $1 \%$. On remarque le rôle important de l'inertie du capteur puisque le rendement n'atteint sa valeur maximale qu'au bout de $2 \mathrm{~h}$ environ.

Le modèle permet d'avoir une bonne connaissance du comportement dynamique du capteur.

\section{Exploitation du modèle.}

Le modèle validé tant en ensoleillement naturel que sous simulateur solaire, permet de quantifier la différence des performances du capteur dans les deux cas et d'évaluer l'erreur commise si les résultats obtenus sous simulateur solaire ne sont pas corrigés pour son utilisation en ambiance naturelle. Il est également possible d'étudier le comportement du capteur en faisant varier des paramètres qu'il est difficile de modifier expérimentalement.

\subsection{COMPARAISONS ENSOLEILLEMENT NATUREL-} SIMULATEUR SOLAIRE. - Le simulateur solaire émet un rayonnement infrarouge important, qui influence les échanges radiatifs de la face avant du capteur. Cette énergie supplémentaire est récupérée partiellement par l'air caloporteur. Les rendements obtenus sous simulateur solaire sont donc supérieurs à ceux qui seraient obtenus à l'extérieur dans les 
Tableau III. - Comparaison ensoleillement naturel-simulateur solaire à débit d'air variable.

[Comparison of collector efficiencies for indoor and outdoor tests as a function of air flow rate.]

\begin{tabular}{|c|c|c|c|c|c|}
\hline Numéro de l'essai & 1 & 2 & 3 & 4 & 5 \\
\hline Débit à la sortie $\left(\mathrm{m}^{3} / \mathrm{h} \cdot \mathrm{m}^{2}\right)$ & 30 & 50 & 70 & 90 & 110 \\
\hline Température de ciel sous simulateur $\left({ }^{\circ} \mathrm{C}\right)$ & 50,5 & 50,0 & 50,1 & 50,3 & 50,1 \\
\hline Température de ciel à l'extérieur $\left({ }^{\circ} \mathrm{C}\right)$ & 5,9 & 4,9 & 5,7 & 7,0 & 6,7 \\
\hline Rendement simulateur solaire ( $\%)$ & 33,2 & 41,4 & 51,5 & 57,1 & 60,0 \\
\hline Rendement ensoleillement naturel $(\%)$ & 29,7 & 37,2 & 46,1 & 51,0 & 53,5 \\
\hline Ecart relatif $(\%)$ & $-10,5$ & $-10,1$ & $-10,5$ & $-10,7$ & $-10,8$ \\
\hline
\end{tabular}

mêmes conditions expérimentales. Ils ne peuvent pas être utilisés sans correction.

Les simulations faites pour le calage du modèle en ensoleillement artificiel ont été reprises, en remplaçant la température de ciel réelle par une température de ciel correspondant à un capteur placé à l'extérieur, obtenue à partir de la relation (10). Dans le tableau III sont donnés les rendements correspondant à des résultats avec un débit variable. Les écarts entre les deux séries de simulations sont importants. Il est donc indispensable de corriger les valeurs expérimentales obtenues en laboratoire avant toute utilisation pour un dimensionnement d'installation par exemple, ce qui est possible à l'aide du modèle décrit.

5.2 ETUde PARAmétriQue. - Pour l'exploitation du modèle, les paramètres de fonctionnement ont les valeurs fixées pour les essais en ensoleillement artificiel avec une température ambiante de $20^{\circ} \mathrm{C}$, une température de ciel de $2,7^{\circ} \mathrm{C}$ et une vitesse du vent de $2 \mathrm{~m} / \mathrm{s}$.

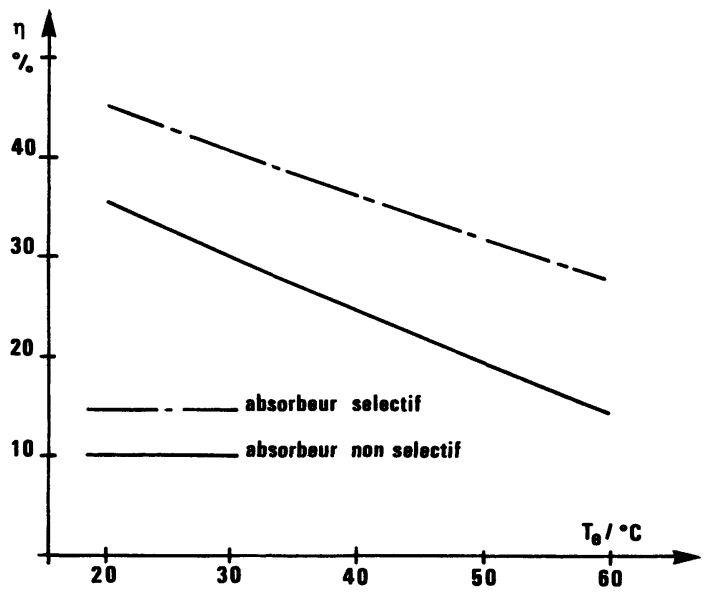

Fig. 6. - Influence de la sélectivité sur le rendement du capteur.

[Collector efficiency as a function of the inlet air temperature for a selective and a non-selective absorber.]
5.2.1 Influence de la sélectivité de l'absorbeur. Sur la figure 6 sont comparés les rendements obtenus pour le capteur expérimental de référence et pour un capteur identique muni d'un absorbeur non sélectif $(\varepsilon=\alpha=0,91)$. Les courbes obtenues s'écartent lorsque la température d'entrée de l'air augmente. L'absorbeur sélectif permet pratiquement de doubler le rendement pour une température d'entrée d'air de $60^{\circ} \mathrm{C}$. Dans un capteur à air, l'absorbeur est toujours beaucoup plus chaud que l'air caloporteur, et les pertes radiatives sont très grandes. L'utilisation d'un absorbeur sélectif permet une diminution très importante de ces pertes.

5.2.2 Influence de la température ambiante. - Le rendement est assez sensible à la température ambiante (Fig. 7) puisqu'il varie d'environ $10 \%$ entre un capteur fonctionnant en hiver et un capteur fonctionnant en été. La diminution du rendement avec la température ambiante provient de l'augmentation des échanges convectifs entre la vitre et l'air ambiant et également de l'augmentation des pertes radiatives, car la température de ciel diminue en même temps que la température ambiante.

5.2.3 Influence du débit de fuite. - L'influence du taux de fuite dépend de la température d'entrée de



Fig. 7. - Influence de la température ambiante sur le rendement du capteur.

[Collector efficiency as a function of the ambient temperature.] 


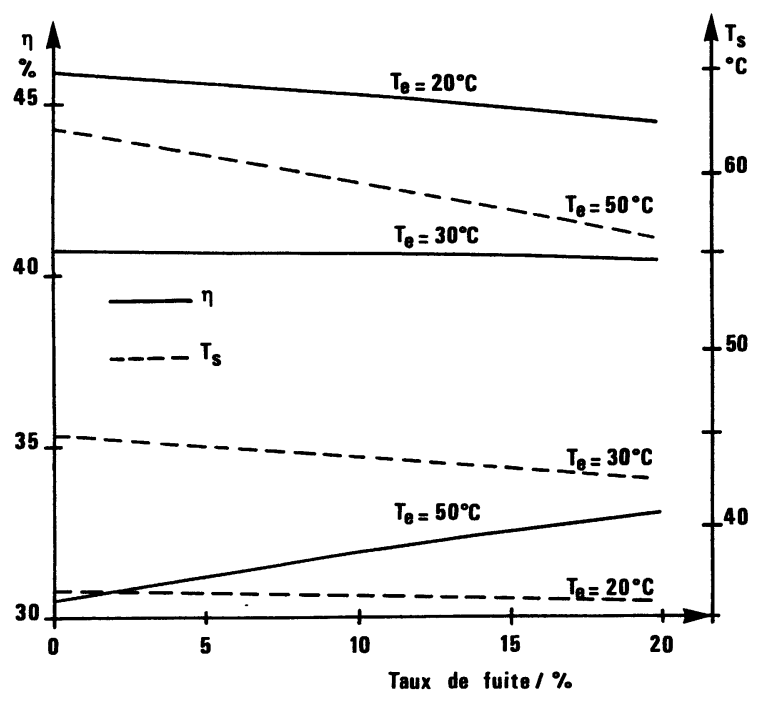

Fig. 8. - Influence du débit de fuite sur le rendement et sur la température de sortie de l'air.

[Collector efficiency and outlet air temperature as a function of air leaks.]

l'air et de la température d'introduction des fuites, c'est-à-dire de la température ambiante (Fig. 8). Les fuites ont deux effets contradictoires :

- d'une part, l'air ambiant introduit dans le capteur, à une température en général inférieure à la température moyenne de l'air dans le capteur, a pour effet d'abaisser les températures des différents éléments constituant le capteur. Par conséquent les pertes thermiques de ce dernier sont réduites et le rendement a tendance à augmenter comme l'a montré Close [11],

- d'autre part, l'air qui pénètre par les fuites ne participe à l'échange avec l'absorbeur que sur une fraction de la longueur de ce dernier et le coefficient d'échange convectif moyen est plus faible que si tout l'air sortant avait léché l'absorbeur sur toute sa longueur.

Pour une température d'entrée élevée $\left(50^{\circ} \mathrm{C}\right)$, le premier effet l'emporte sur le deuxième et le rendement augmente avec le taux de fuite. Si la température d'entrée est basse $\left(20^{\circ} \mathrm{C}\right)$, le deuxième effet est prépondérant et le rendement diminue avec le taux de fuite. Pour une température d'entrée de $30^{\circ} \mathrm{C}$, les deux effets se compensent et le rendement est quasiment indépendant du taux de fuite.

Cette analyse semble prouver que plus les fuites sont importantes, meilleur est le rendement du capteur, dès qu'il fonctionne au-delà d'un certain niveau de température d'entrée. Cette conclusion doit être modifiée si on considère les variations de la température de sortie de l'air en fonction du taux de fuite (Fig. 8). Dans tous les cas, celle-ci diminue lorsque le débit de fuite augmente, puisqu'une quantité croissante d'air froid est introduite dans le capteur. Pour garder un niveau de température de sortie suffisant, ce qui permet une bonne utilisation de l'énergie récupérée par le capteur, par exemple pour du stockage, il est nécessaire que celui-ci ait la meilleure étanchéité possible.

\section{Conclusion.}

Le comportement de capteurs solaires à air a été étudié en ensoleillement naturel et en ensoleillement artificiel de façon à mieux maîtriser les paramètres dont dépend le rendement. Il est à noter que ce type de capteurs, montés en toiture, a une inertie relativement importante qui entraîne une faible valeur du rendement du capteur en début de journée, et une valeur plus élevée en fin de journée. Alors que le débit d'air dans le capteur et la température d'entrée ont une grande influence sur le rendement, par contre l'inclinaison du capteur et l'éclairement influent peu.

$\mathrm{La}$ modélisation en régime dynamique rend compte du comportement du capteur avec une bonne précision. Elle confirme le rôle important de l'inertie du capteur. Elle a permis de mettre en évidence l'intérêt des absorbeurs sélectifs pour les capteurs à air, qui permettent une amélioration considérable des rendements lorsque la température d'entrée de l'air est élevée. Les fuites, inévitables dans les capteurs à air, diminuent la température de sortie de l'air, mais améliorent dans certains cas le rendement. Le modèle permet également de corriger les mesures faites sous simulateur ; ainsi une surestimation du rendement en ensoleillement artificiel de l'ordre de $10 \%$ a été mise en évidence.

\section{Bibliographie}

[1] NiLeS, P. W., Design and performance of an air collector for industrial crop dehydration. Solar Energy 20 (1978) 19-23.

[2] Gupta, G. L., GARG, H. P., Performance studies on solar air heaters. Solar energy 11 (1967) 25-31.

[3] LETZ, T., Modélisation et dimensionnement économique d'un système de chauffage domestique biénergie (solaire-électrique). Expérimentation de capteurs à air. Thèse de Doctorat, I.N.S.A. Lyon, (1985).

[4] Kreith, F., Kreider, J. F., Principles of solar engineering (Mc Graw Hill) 1978.

[5] KLEIN, S. A., Calculation of flat-plate collector coefficients. Solar Energy 17 (1975) 79-80.

[6] Devin, B., Analyse physique et expérimentale des capteurs plans en régime statique. Rapport C.E.A., 60 p. (1977).

[7] Granier, P., DAguenet, M., Les insolateurs à air multipasses. Revue Int. Héliotech., 21-25 (1er semestre 1981).

[8] Granier, P., Les insolateurs à air multipasses. Thèse de Docteur Ingénieur, Perpignan (1967).

[9] Boudarel, R., DELMAS, J., GUICHET, P., Commande optimale des processus. Concepts fondamentaux de l'automatique (Dunod) 1967.

[10] PePIN-Bosc, M. H., Le rayonnement atmosphérique. Coll. Météorologie et énergies renouvelables, A.F.M.E., Valbonne, 13-16 Mars, 1984.

[11] Close, D. J., Yusoff, M. B., The effects of air leaks on solar air collector behaviour. Solar Energy 20 (1978) 459-463. 